\title{
On Chronophysiomics: Prospects to Optimize Appetite and Health
}

\author{
Nikkhah $A^{*}$ \\ Department of Animal Sciences Faculty of Agricultural Sciences, University of Zanjan
}

*Corresponding author: Nikkhah A, Chief Highly Distinguished Professor, Department of Animal Sciences Faculty of Agricultural Sciences, University of Zanjan, Zanjan 313-45195 Iran, Tel: +98-241-5152801, Fax: +98241-5283202, E-mail: nikkhah@znu.ac.ir

Citation: Nikkhah A (2014) On Chronophysiomics: Prospects to Optimize Appetite and Health. SAJ Biotechnol 1: 103. doi: $10.18875 / 2375-6713.1 .103$

Article history: Received: 28 May 2014, Accepted: 16 July 2014, Published: 18 July 2014

This editorial describes evolving chronophysiomical mediations of appetite and food intake (AFI). Chronophysiomics is a multiscience involving integrated chronophysiology and functional omics biotechnologies. Chronophysiology as an evolutionary interscience has enabled humans and animals to better cope with the changing environment from the mother nature towards modernity. This trend continues to evolve into postmodernity. With the recent discoveries on timing of eating and feeding orchestrating circadian and postprandial nutrient intake and metabolic rhythms in animal models [1-7], time has already come to contemplate chronophysiology as a key regulator of AFI. Omics biotechnologies (e.g., genomics, proteomics, lipidomics, transcriptomics, and metabolomics) provide new tools to further our perceptions of possible mechanisms underlying such regulations. This is important since AFI affects directly or circuitously risks of health issues such as obesity, diabetes mellitus, blood pressure and cardiovascular diseases.

Chronophysiomics has been a key missing postmodern biotechnology in realistic modeling of AFI regulation. The shortcoming gains increasing thoughts given that accurate and reliable appetite and nutrient intake predictions are essential to consistent meeting of nutrient requirements and, thus, to healthy human life. In farm animal ruminant models, altered evolutionary circadian and postprandial rhythms in grazing, chewing, rumen fermentation, and peripheral substrate assimilation in response to shifting timing of eating have been discovered. These may help develop innovative strategies to improve appetite prediction accuracy and, thus, to help control appetite for durable health.

Inspired by the nature, ruminants have evolved to ruminate habitually overnight and graze during day. Accordingly, rumen fermentation and peripheral metabolism have developed specialized circadian rhythms [2,3]. These evolutionary rhythms in eating behavior and metabolism have generated circannual, seasonal, and circadian rhythms in endocrinology. The drastic changes in production systems due to modernization have altered such natural eating, ruminating and rumen rhythms. Therefore, it has become vital to preserve synchronies between external cues and ruminant internal state $[2,4]$.

Most recent findings suggest altered postfeeding AFI rhythms of nongrazing cows by shifting feeding timing [1,5]. Eating rate and AFI, especially shortly postfeeding, have increased by night instead of day feeding. As a result, rhythms of circadian and postprandial rumen fermentation and intermediary metabolism have changed [6,7]. Timing of feeding/eating as a key external manager, thus, determines the rate and extent of AFI and splnachnoperipheral metabolism. This animal model data imply that timing of eating may be used as a feasible strategy to manipulate and optimize appetite and cell health. However, conclusive data on omics will need to be generated to enable drawing pathways through which timing of eating (or chronophyiomics) orchestrates appetite and intermediary metabolism and health.

The impacts of chronophysiomical management strategies (e.g., eating timing) must be quantified for inclusion in appetite and nutrient intake prediction under varying physiological states and life styles. This innovative biotechnological revelation will help enhance health-promoting functions of foods for improved global health and life quality.

\section{Acknowledgment}

The Ministry of Science, Research and Technology, and University of Zanjan, Iran, are thankfully acknowledged for supporting the author's global programs of optimizing the new millennium science edification.

\section{References}

1. Nikkhah A (2013) Chronophysiology of ruminant feeding behavior and metabolism: an evolutionary review. Biol Rhythm Res 44: $197-218$. 
2. Nikkhah A (2011) Bioscience of ruminant intake evolution: feeding time models. Adv Biosci Biotechnol 2: 271-4.

3. Taweel HZ, Tas BM, Dijkstra J, Tamminga S (2004) Intake regulation and grazing behavior of dairy cows under continuous stocking J Dairy Sci 87: $3417-27$.

4. Nikkhah A (2011) Ruminant chronophysiological management: an emerging bioscience. Open Access Anim Physiol 3: 9-12.

5. Nikkhah A (2011) Science of eating time: A novel chronophysiological approach to optimize glucose-insulin dynamics and health. J Diab Mellit 2: 8-11.

6. Nikkhah A, Furedi C, Kennedy A, Wittenberg K, Scott SL, et al. (2010) Feed delivery at $2100 \mathrm{~h}$ vs. $0900 \mathrm{~h}$ for lactating dairy cows. Can J Anim Sci $91: 113-22$.

7. Nikkhah A (2012) Timing of feed presentation entrains periprandial rhythms of energy metabolism indicators in once-daily fed lactating cows. Biol Rhythm Res 43: 651-61. 